\section{(6) OPEN ACCESS}

\title{
Improvements in ECG accuracy for diagnosis of left ventricular hypertrophy in obesity
}

\author{
Oliver J Rider, ${ }^{1}$ Ntobeko Ntusi, ${ }^{1,2}$ Sacha C Bull, ${ }^{1}$ Richard Nethononda, ${ }^{3}$ \\ Vanessa Ferreira, ${ }^{1}$ Cameron J Holloway, ${ }^{4}$ David Holdsworth, ${ }^{1}$ Masliza Mahmod, ${ }^{1}$ \\ Jennifer J Rayner, ${ }_{1}^{1}$ Rajarshi Banerjee, ${ }^{1}$ Saul Myerson, ${ }^{1}$ Hugh Watkins, ${ }^{1}$ \\ Stefan Neubauer ${ }^{1}$
}

${ }^{1}$ Radcliffe Department of Medicine, Division of Cardiovascular Medicine and University of Oxford Centre for Clinical Magnetic Resonance Research, University of Oxford, Oxford, UK

${ }^{2}$ Division of Cardiology, Department of Medicine Research, University of Capetown, South Africa ${ }^{3}$ Chris Hani Baragwanath Hospital, Soweto \& University of Witwatersrand, Johannesburg, South Africa ${ }^{4}$ St Vincent's Hospital, Sydney, Australia

\section{Correspondence to} Dr Oliver J Rider, Radcliffe Department of Medicine, Division of Cardiovascular Medicine, OCMR, Level 0, John Radcliffe Hospital, Oxford OX3 9DU, UK; oliver.rider@cardiov.ox.ac.uk

Received 18 December 2015 Revised 14 April 2016 Accepted 25 April 2016 Published Online First 2 August 2016

\begin{abstract}
Objectives The electrocardiogram (ECG) is the most commonly used tool to screen for left ventricular hypertrophy (LVH), and yet current diagnostic criteria are insensitive in modern increasingly overweight society. We propose a simple adjustment to improve diagnostic accuracy in different body weights and improve the sensitivity of this universally available technique. Methods Overall, 1295 participants were included821 with a wide range of body mass index (BMl $17.1-53.3 \mathrm{~kg} / \mathrm{m}^{2}$ ) initially underwent cardiac magnetic resonance evaluation of anatomical left ventricular (LV) axis, LV mass and 12-lead surface ECG in order to generate an adjustment factor applied to the SokolowLyon criteria. This factor was then validated in a second cohort $(n=520$, BMI 15.9-63.2 kg/m²).
\end{abstract}

Results When matched for LV mass, the combination of leftward anatomical axis deviation and increased BMI resulted in a reduction of the Sokolow-Lyon index, by $4 \mathrm{~mm}$ in overweight and $8 \mathrm{~mm}$ in obesity. After adjusting for this in the initial cohort, the sensitivity of the Sokolow-Lyon index increased (overweight: $12.8 \%$ to $30.8 \%$, obese: $3.1 \%$ to $27.2 \%$ ) approaching that seen in normal weight (37.8\%). Similar results were achieved in the validation cohort (specificity increased in overweight: $8.3 \%$ to $39.1 \%$, obese: $9.4 \%$ to $25.0 \%$ ) again approaching normal weight (39.0\%). Importantly, specificity remained excellent (>93.1\%).

Conclusions Adjusting the Sokolow-Lyon index for $\mathrm{BMl}$ (overweight $+4 \mathrm{~mm}$, obesity $+8 \mathrm{~mm}$ ) improves the diagnostic accuracy for detecting LVH. As the ECG, worldwide, remains the most widely used screening tool for LVH, implementing these findings should translate into significant clinical benefit.

\section{INTRODUCTION}

As electrocardiographic determination of left ventricular hypertrophy (LVH) is not costly, is simple to perform and yields indices linked to mortality, ${ }^{12}$ it is widely used in clinical practice and appears in international guidelines. ${ }^{3} 4$ As a result, the electrocardiogram (ECG) remains, worldwide, the most commonly used screening tool.

The two most commonly used ECG criteria are the Sokolow-Lyon index 5 and the Cornell criteria. Despite their routine clinical use, the diagnostic accuracy of these surface ECG criteria is limited, with poor sensitivity for the detection of LVH. ${ }^{6} 7$ Indeed, the original authors of the Sokolow-Lyon index report only a $32 \%$ sensitivity, making its uptake into clinical practice and its continued use surprising. Obesity, which is now pandemic in the Western world, results in three distinct processes that affect the surface ECG-lateral displacement of the anatomical left ventricular (LV) axis, increased chest wall fat and increased pericardial fat mass, all of which decrease voltage amplitude on the ECG. ${ }^{8-10}$ This reduces the diagnostic sensitivity of the surface ECG even further and is likely to render ECG criteria generated in a society with a much lower body mass index (BMI) $\left(\sim 20 \mathrm{~kg} / \mathrm{m}^{2}\right.$ in 1949$)^{11}$ to be obsolete in the modern age of obesity, where LVH is increasingly seen. ${ }^{12}$

Although previous attempts have been made at improving the diagnostic accuracy of the ECG by adjusting for body habitus, they have either relied on echocardiographic determination of LV mass, ${ }^{13}{ }^{14}$ which is severely limited in obesity, or have not taken into account the anatomical LV axis deviation that accompanies obesity. ${ }^{15}$ Furthermore, although all report diagnostic performance improvement, these studies all implemented complex statistical modelling to derive adjustment formula that could be applied to the ECG to adjust for obesity. ${ }^{6}$ Hence, they are not practical to perform as a quick screening tool and have failed to enter routine clinical practice.

The aim of this study was to develop a simple BMI-based adjustment factor for the ECG that could be used in everyday clinical practice to improve diagnostic accuracy of the detection of LVH in the modern population, where obesity is increasingly prevalent.

\section{METHODS}

\section{Initial cohort population}

All research data acquisition was approved by the local research ethics committee and informed written consent was obtained from each participant. Participants were recruited from the Oxfordshire population to studies within the Oxford Centre for Clinical Magnetic Resonance Research (OCMR) between 2005 and 2012. A flow chart of subjects through phase I of the study is shown in figure 1 . All participants were subject to the same exclusion criteria-pregnancy, under 18 years of age, claustrophobia and metallic foreign body. Due to effects on ECG voltage amplitude, subjects with complete left or right bundle branch block (LBBB or RBBB),
To cite: Rider OJ, Ntusi $\mathrm{N}$ Bull SC, et al. Heart

1566 


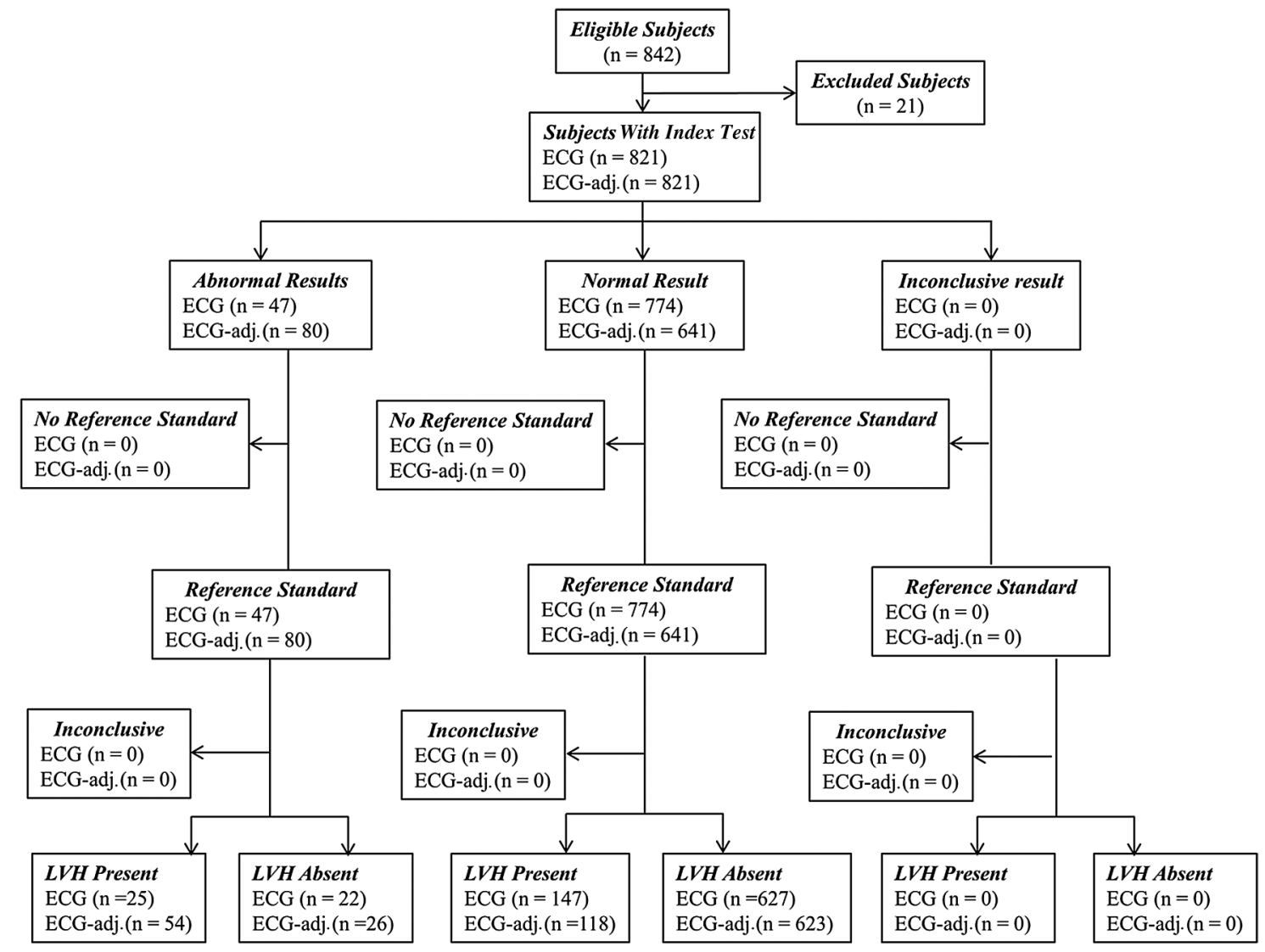

Figure 1 Flow chart of subjects through the study. ECG, electrocardiogram; LVH, left ventricular hypertrophy.

chronic obstructive pulmonary disease, imaging evidence of myocardial infarction, hypertrophic cardiomyopathy or significant $(>1 \mathrm{~cm})$ pericardial effusion were excluded. Of the identified 842 subjects fulfilling inclusion criteria, 21 were excluded from final analysis (15 with either LBBB or RBBB, 4 without Half Fourier Acquisition Single shot Turbo spin Echo (HASTE) imaging and 2 with non-diagnostic cardiovascular magnetic resonance (CMR) quality). A retrospective analysis of the ECGs and CMR scans was performed on the remaining 821 participants $\left({ }^{*} n=450\right.$, $\$ n=371$, aged $19-87$ years) across a wide range of BMI (17.1-53.3 kg/m²). The group was predominantly Caucasian in origin.

\section{Validation cohort}

All 520 adult participants for the validation cohort were recruited in the same fashion, with additional recruitment from the Division of Cardiology, Department of Medicine Research, University of Capetown, South Africa. To maximise generalisability of the study, within this cohort, 9.3\% were African (normal weight $60 \%$, overweight $19 \%$, obese $21 \%$ ) and $90.7 \%$ were Caucasian. Inclusion and exclusion criteria were as described above. A retrospective analysis was performed on these 520 adult participants $\left({ }^{*} \mathrm{n}=228\right.$, BMI $\left.15.9-63.2 \mathrm{~kg} / \mathrm{m}^{2}\right)$.

\section{Anthropometric data}

Height and weight were measured using a digital station (Seca, UK) and used to calculate BMI. Subjects were grouped according to World Health Organisation BMI categories: normal $\left(18.5-24.9 \mathrm{~kg} / \mathrm{m}^{2}\right)$, overweight $\left(25.0-29.9 \mathrm{~kg} / \mathrm{m}^{2}\right)$ or obese $\left(>30.0 \mathrm{~kg} / \mathrm{m}^{2}\right.$ ). Blood pressure was recorded (DINAMAP-1846SX, Critikon Corp.) (table 1).

\section{MAGNETIC RESONANCE IMAGING LV axis}

The anatomical axis of the LV was determined in the coronal and sagittal planes using multiplanar reconstruction of transverse thoracic HASTE images within cmr42 (Circle Cardiovascular Imaging, Calgary, Canada). The anatomical LV axis was defined as the plane between the centre of the mitral valve and the LV apex determined on both the horizontal and vertical long axis views. The $\mathrm{LV}$ axis in both planes (degrees $0^{\circ}$ horizontal) was determined using a commercially available screen protractor (Screen Protractor 4.0, Iconico, New York, USA). Representative images of this process are shown in figure 2.

\section{LV mass and analysis}

All imaging was ECG gated and acquired during breath-hold. In brief, a short axis stack of LV images was acquired (slice thickness $7 \mathrm{~mm}$, gap $3 \mathrm{~mm}$ ) using a steady-state free precession sequence (echo time of $1.5 \mathrm{~ms}$, repetition time of $3.0 \mathrm{~ms}$, temporal resolution of $47.84 \mathrm{~ms}$, flip angle of $60^{\circ}$ ) as previously described. ${ }^{16-18}$ Image analysis for LV mass was performed using cmr42 by a single experienced operator with $>9$ years of CMR experience (OJR), as previously described. ${ }^{19} \mathrm{LVH}$ was defined as $>2 \mathrm{SD}$ higher than the published mean of the Oxfordshire population from which the study sample was taken $(>165 \mathrm{~g}$ in men, $>150 \mathrm{~g}$ in women). ${ }^{20}$ On repeat analysis of 20 scans, intraobserver variability was determined to be $6.1 \%$. This is in keeping with previous reports. ${ }^{20}$

\section{ECG recording and analysis}

A standard 12-lead ECG was performed in all participants (Fukuda Denshi Systems, UK) on the same day as the CMR. 
Table 1 The relationship between body mass index (BMI), electrocardiogram left ventricular hypertrophy criteria, CMR left ventricular (LV) mass and anatomical LV axis in the initial and validation cohorts

\begin{tabular}{|c|c|c|c|}
\hline Initial cohort $(n=821)$ & $\begin{array}{l}\text { Normal weight } \\
n=263\end{array}$ & $\begin{array}{l}\text { Overweight } \\
n=296\end{array}$ & $\begin{array}{l}\text { Obese } \\
n=262\end{array}$ \\
\hline Age (years) & $50(15)$ & $55(15)$ & $54(14)$ \\
\hline Height $(\mathrm{cm})$ & $171(9)$ & $171(10)$ & $168(9)$ \\
\hline Systolic blood pressure $(\mathrm{mm} \mathrm{Hg})$ & $126(17)$ & $136(19)$ & $135(19)$ \\
\hline Diastolic blood pressure $(\mathrm{mm} \mathrm{Hg})$ & $75(9)$ & $80(10)$ & $81(10)$ \\
\hline BMI $\left(\mathrm{kg} / \mathrm{m}^{2}\right)$ & $23(2)$ & $27(1)$ & $35(6)$ \\
\hline Frontal plane axis $\left(^{\circ}\right)$ & $45(12)$ & $38(13)$ & $28(13)$ \\
\hline Sagittal plane axis $\left({ }^{\circ}\right)$ & $137(12)$ & $147(12)$ & $154(11)$ \\
\hline LV mass $(\mathrm{g})$ & $119(41)$ & $137(46)$ & $141(42)$ \\
\hline Sokolow-Lyon voltage (mm) & $23(8)$ & $21(7)$ & $19(6)$ \\
\hline Cornell voltage $(\mathrm{mm})$ & $13(7)$ & $14(8)$ & $14(6)$ \\
\hline Validation cohort $(n=520)$ & $\begin{array}{l}\text { Normal weight } \\
n=206\end{array}$ & $\begin{array}{l}\text { Overweight } \\
\mathrm{n}=169\end{array}$ & $\begin{array}{l}\text { Obese } \\
n=145\end{array}$ \\
\hline Age (years) & $52(13)$ & $52(11)$ & $54(11)$ \\
\hline Height $(\mathrm{cm})$ & $170(9)$ & $172(9)$ & $171(10)$ \\
\hline $\mathrm{BMI}\left(\mathrm{kg} / \mathrm{m}^{2}\right)$ & $22(2)$ & $27(1)$ & $34(4)$ \\
\hline LV mass $(\mathrm{g})$ & $100(31)$ & $113(39)$ & $121(40)$ \\
\hline Sokolow-Lyon voltage (mm) & $21(8)$ & $19(9)$ & $17(6)$ \\
\hline
\end{tabular}

ECG measurements were performed manually by two experienced readers with $>10$ years of experience in ECG interpretation (OJR \& DH). The analysis was blinded to participant's BMI and LV mass. The following ECG criteria were examined as reference standards: Sokolow-Lyon voltage (S-wave $\mathrm{V}_{1}+\mathrm{R}$-wave lead $\mathrm{V}_{5}$ or $\left.\mathrm{V}_{6}\right)^{5}$ and Cornell voltage (R-wave aVL $+\mathrm{S}$-wave $\left.\mathrm{V}_{3}\right)^{21}$ Measurements were taken to the nearest $0.1 \mathrm{mV}$. On repeat analysis (25 ECGs), interobserver variability for ECG analysis was $<0.1 \mathrm{mV}$.

\section{Statistical analysis}

All statistics were analysed using commercial software (SPSS V.20, Chicago, Illinois, USA). All data were normally distributed according to Kolmogorov-Smirnov testing and the results are presented as mean (SD). Group comparison data were analysed using ANOVA with Bonferroni correction. Analysis of the effects of BMI and LV axis on ECG criteria was performed using Analysis of Covariance (ANCOVA) with post hoc Bonferroni correction. Homogeneity of variance was tested with Levene's tests and visually with residual plots. Sensitivity and values were calculated before and after correction for BMI and presented with 95\% confidence intervals (CIs). Positive and negative predictive values and diagnostic accuracy are also calculated. To compare the diagnostic accuracy improvements, $\chi^{2}$ and McNemar testing were performed. Data are presented as receiver operating characteristic (ROC) curves and overall diagnostic accuracy was interrogated using the Youden index. The values of $p<0.05$ were considered as statistically significant.

\section{RESULTS}

\section{CMR-derived and ECG-derived LVH for the initial study} cohort

Of the 821 participants, 172 had CMR-defined LVH (21.0\%). Despite an increase in LV mass with obesity $(22 \mathrm{~g}, \mathrm{p}<0.001)$, Sokolow-Lyon index voltage criteria decreased by $4 \mathrm{~mm}$ $(p<0.001)$ and Cornell criteria did not increase $(p>0.99$, table 1). This suggests that both criteria are significantly affected by obesity and that the Sokolow-Lyon would be more suitable for a BMI adjustment factor.

\section{The effect of obesity on diagnostic accuracy of ECG criteria}

The Sokolow-Lyon criteria had poor sensitivity of $14.7 \%$ (CI $10.0 \%$ to $20.8 \%$ ) but excellent specificity of $96.7 \%(95.1 \%$ to 97.8\%) for the detection of CMR-determined LVH. Overall, diagnostic accuracy was very poor; ROC area under curve (AUC) was 0.55 , Youden index was 0.11 (CI 0.05 to 0.16 ), positive predictive value was 0.52 (CI 0.37 to 0.67 ) and negative predictive value was 0.81 (CI 0.78 to 0.83 ) (figure 3 ).

However, this diagnostic performance was also too dependent on body habitus. The diagnostic sensitivity of the Sokolow-Lyon index is reduced with increasing BMI (normal 37.8\% (CI 24.1\% to $53.9 \%$ ), overweight $12.8 \%$ (CI $6.9 \%$ to $22.7 \%$ ) and obesity $3.1 \%$ (CI $0.4 \%$ to $10.8 \%$ ), $\chi^{2} 12.6, p=0.02$ ). In contrast, the specificity was seen to increase with increasing obesity (normal weight $92.9 \%$ (88.8\% to $95.6 \%$ ), overweight $98.2 \%$ (95.5\% to $99.3 \%$ ) and obesity $99.0 \%$ (96.4\% to $99.9 \%)$, table 2). As a result, in obesity, where the decrease in voltage amplitude greatly limits the ability to generate a positive result, the Sokolov-Lyon criterion performs extremely poorly (ROC AUC 0.51 , Youden index 0.02 (CI -0.01 to 0.06$)$ ).

The Cornell criteria had comparable diagnostic performance with the Sokolow-Lyon criteria with $14.8 \%$ (10.4\% to $20.1 \%)$ sensitivity and $96.7 \%$ (94.9\% to $97.8 \%$ ) specificity for detecting LVH. Again, a fall in sensitivity was seen with obesity from $21.1 \%(11.1 \%$ to $36.4 \%)$ to $11.9 \%(6.2 \%$ to $21.8 \%)$ with no change in specificity (table 2 ).

\section{Defining the effect of obesity on LV anatomical axis}

When considering the whole study cohort, the incidence of a more leftward anatomical axis (defined as the lowest coronal axis tertile) increased from $13 \%$ in normal weight to $58 \%$ in the obesity, with leftward displacement of the LV axis in the coronal plane and superior displacement in the sagittal plane by on average $17^{\circ}$ (both $\mathrm{p}<0.001$ ).

\section{The effect of anatomical axis deviation on ECG voltage criteria}

In order to determine the effect of an increasing leftward anatomical axis on ECG voltage criteria, subjects were matched for both LV mass and BMI. This showed that, for a given LV mass and BMI, a leftward deviation of the LV anatomical axis was associated with a significant decrease in Sokolow-Lyon amplitude (by 3-5 mm, p $<0.05$ all analyses). Similar results were seen using ANCOVA analysis investigating the effects of LV axis deviation on ECG criteria (covariates in model evaluated at LV mass $133 \mathrm{~g}$, BMI $28.2 \mathrm{~kg} / \mathrm{m}^{2}$ (mean values for the cohort)) with reducing Sokolow-Lyon voltage with increasing lateral axis displacement (lowest tertile $22.6 \mathrm{~mm}$, middle tertile $21.5 \mathrm{~mm}$, obese $18.9 \mathrm{~mm}$, all post hoc comparisons $\mathrm{p}<0.05)$. There were no significant changes in Cornell criteria ( $p>0.99$ all analyses).

\section{The effect of obesity on ECG voltage criteria}

In order to determine the effect of obesity per se, subjects were matched for both LV mass and LV anatomical axis. This showed that, for any given LV mass and anatomical orientation, increasing BMI is associated with a significant decrease in SokolowLyon index (overweight by $2-4 \mathrm{~mm}$, obese by $3-5 \mathrm{~mm}, \mathrm{p}<0.05$ all analyses). Similar results were seen using ANCOVA analysis investigating the effects of obesity on ECG criteria (covariates in 
Male

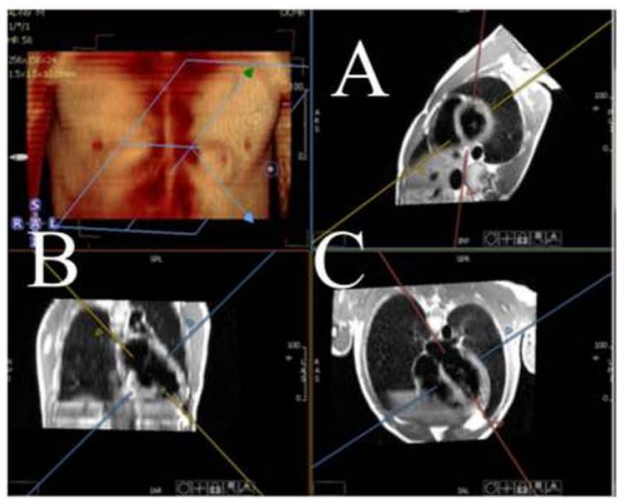

Female

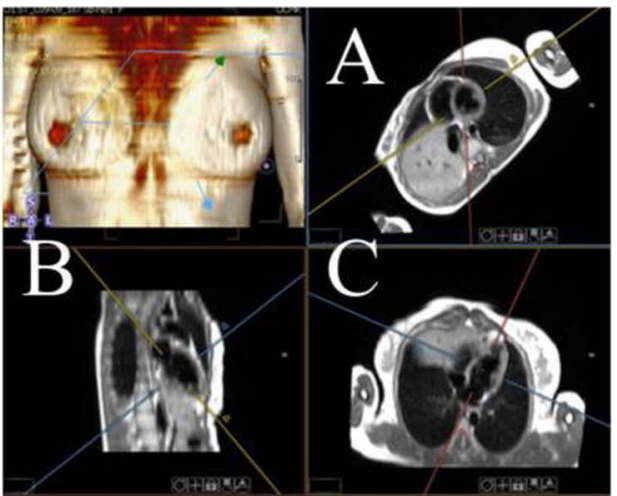

Figure 2 Defining the coronal anatomical left ventricular axis using multiplanar reconstruction. Images show the coronal axis in the (A) short axis, (B) vertical long axis and (C) horizontal axis views.

model evaluated at LV mass $133 \mathrm{~g}$, LV coronal axis $37^{\circ}$ (mean values for the cohort)) with reducing Sokolow-Lyon index with increasing BMI (normal $23.0 \mathrm{~mm}$, overweight $20.6 \mathrm{~mm}$, obese $19.5 \mathrm{~mm}$, all post hoc comparisons $\mathrm{p}<0.05$ ).

\section{Defining a BMI adjustment factor for the Sokolow-Lyon index}

The adjustment factors for the overweight and obese cohorts were calculated as the maximum sum of the effects of both BMI and leftward anatomical LV axis deviation. This showed that when matched for $\mathrm{LV}$ mass, on average, a $4 \mathrm{~mm}$ reduction in ECG voltage amplitude was observed in overweight and an $8 \mathrm{~mm}$ reduction was observed with obesity. As no significant change in Cornell criteria was observed with increasing BMI, no adjustment factor was calculated.

\section{The effect of adjusting ECG criteria on diagnostic} performance in the initial cohort

When adjusting the Sokolow-Lyon index (overweight $+4 \mathrm{~mm}$, obesity $+8 \mathrm{~mm}$ ) for the entire initial study group, the sensitivity increased from $14.7 \%(10.0 \%$ to $20.8 \%)$ to $31.4 \%$ (24.9\% to $38.7 \%)(\mathrm{p}<0.001$, table 2$)$. The largest gain in sensitivity was observed in the obese group from $3.1 . \%(0.4 \%$ to $10.8 \%)$ to $27.2 \%(18.0 \%$ to $39.0 \%)$, with lower, but substantial gains observed in the overweight group from $12.8 \%$ to $30.8 \%$ (table 2 all $\mathrm{p}<0.001)$. Notably, specificity remained high at $>96.9 \%$, even
Figure 3 The effect of obesity and leftward axis deviation on electrocardiogram (ECG) voltage criteria. (A) Clear left ventricular hypertrophy (LVH) in obesity with leftward axis and negative SokolowLyon criteria for LVH, which becomes positive only when adjusted for body mass index (BMI) (by $+8 \mathrm{~mm}$ ) and (B) clear LVH in a normal weight participant with normal left ventricular (LV) anatomical axis and positive Sokolow-Lyon criteria for LVH. IVSd, Intraventricular Septum in Diastole.
A

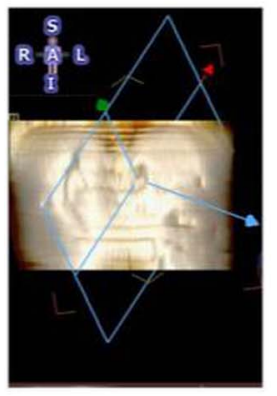

Coronal Axis $19.2^{\circ}$

B

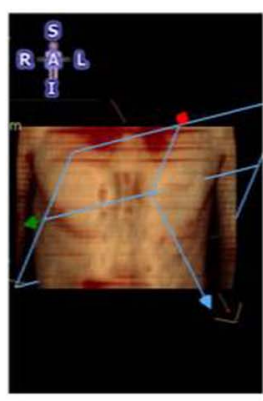

Coronal Axis $62.2^{\circ}$
BMI $31.9 \mathrm{~kg} / \mathrm{m}^{2}$
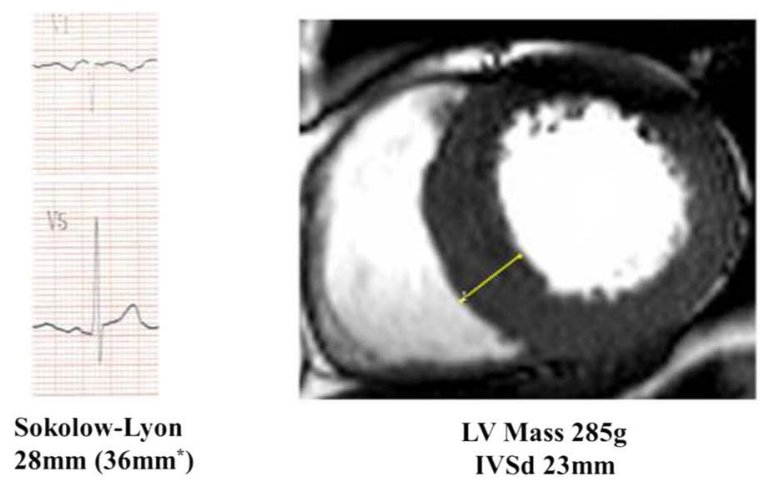

LV Mass 285g

IVSd $23 \mathrm{~mm}$

BMI $21.5 \mathrm{~kg} / \mathrm{m}^{2}$

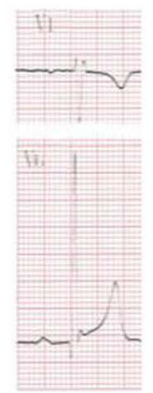

Sokolow-Lyon $45 \mathrm{~mm}$

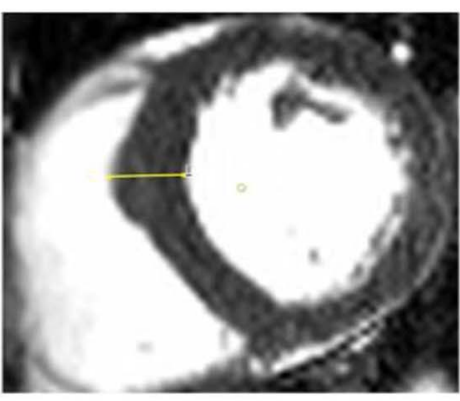

LV Mass 214g IVSd $20 \mathrm{~mm}$ 
Table 2 Diagnostic accuracy of unadjusted and body mass index-adjusted electrocardiogram criteria for anatomic

\begin{tabular}{|c|c|c|c|c|c|}
\hline & Sensitivity (\%) & Specificity (\%) & Positive predictive value (\%) & Negative predictive valve (\%) & Diagnostic accuracy (\%) \\
\hline \multicolumn{6}{|c|}{ Initial cohort $(n=821)$, Sokolow-Lyon index $>35 \mathrm{~mm}\left({ }^{*}=p<0.05\right.$ for adjusted vs non-adjusted) } \\
\hline \multicolumn{6}{|c|}{ Total } \\
\hline Unadjusted & $14.7(10.0$ to 20.8$)$ & $96.7(95.1$ to $97 \cdot 8)$ & 53.2 (38.1 to 67.9$)$ & $81.4(78.5$ to 84.1$)$ & 79.8 \\
\hline Adjusted & $31.4(24.9 \text { to } 38.7)^{*}$ & $94.7(92 \cdot 7 \text { to } 96 \cdot 1)^{*}$ & 66.25 (54.8 to 76.5$)$ & $84.4(81.4$ to 86.7$)$ & 82.6 \\
\hline \multicolumn{6}{|l|}{ Normal weight } \\
\hline Unadjusted & $37.8(24 \cdot 1$ to 53.9$)$ & $92.9(88.8$ to $95 \cdot 6)$ & 46.67 (28.3 to 65.7$)$ & 97.1 (95.7 to 98.2$)$ & 85.5 \\
\hline \multicolumn{6}{|l|}{ Overweight } \\
\hline Unadjusted & $12 \cdot 8(6.9$ to $22 \cdot 7)$ & $98 \cdot 2(95 \cdot 5$ to $99 \cdot 3)$ & 69.2 (38.6 to 90.9$)$ & 78.6 (73.4 to 83.2$)$ & 78.2 \\
\hline Adjusted & $30 \cdot 8(20 \cdot 2 \text { to } 43 \cdot 3)^{*}$ & $96.9(93.8 \text { to } 98 \cdot 8)^{*}$ & 75.0 (55.1 to 89.3$)$ & 82.5 (77.4 to 86.6 ) & 81.8 \\
\hline \multicolumn{6}{|l|}{ Obese } \\
\hline Unadjusted & $3.1(0.4$ to 10.8$)$ & 99.0 (96.4 to 99.9$)$ & 50.0 (6.76 to 93.24$)$ & 76.0 (70.3 to 81.1$)$ & 75.3 \\
\hline Adjusted & $27.2(18.0 \text { to } 39.0)^{*}$ & $97.9(94.8 \text { to } 99.2)^{*}$ & 80.9 (58.1 to 94.6$)$ & 81.1 (75.5 to 85.9$)$ & 81.4 \\
\hline \multicolumn{6}{|c|}{ Validation cohort $(n=520)$, Sokolow-Lyon index $>35 \mathrm{~mm}\left({ }^{*}=p<0.05\right.$ for adjusted vs non-adjusted) } \\
\hline Unadjusted & $17.9(8.9$ to 28.7$)$ & $97.6(95 \cdot 7$ to $98 \cdot 8)$ & 50.0 (28.2 to 71.8$)$ & 89.4 (86.3 to 91.9 ) & 87.7 \\
\hline Adjusted & $28.1(17.6 \text { to } 40 \cdot 8)^{*}$ & $95.2(92.8 \text { to } 96.9)^{*}$ & 45.0 (29.3 to 61.6$)$ & 90.4 (87.4 to 92.3 ) & 86.9 \\
\hline \multicolumn{6}{|l|}{ Normal weight } \\
\hline Unadjusted & $39.0(13.9$ to 68.2$)$ & $95.4(91.5$ to 97.8$)$ & 35.7 (12.8 to 64.9$)$ & 95.9 (92.1 to 98.2$)$ & 91.9 \\
\hline \multicolumn{6}{|l|}{ Overweight } \\
\hline Unadjusted & $8.3(1.1$ to 28.0$)$ & $98.6(95 \cdot 1$ to 99.8$)$ & 50.1 (6.8 to 93.0$)$ & 87.1 (81.0 to 91.9 ) & 86.2 \\
\hline Adjusted & $39.1(19.7 \text { to } 61.5)^{*}$ & $93.1(87.6 \text { to } 96.6)^{*}$ & 47.4 (24.5 to 71.1$)$ & 90.1 (84.5 to 94.7 ) & 85.6 \\
\hline \multicolumn{6}{|l|}{ Obese } \\
\hline Unadjusted & $9.4(1.9$ to $25 \cdot 0)$ & 99.1 (95.0 to 99.9$)$ & 75.0 (19.4 to 99.4$)$ & 79.0 (71.2 to 85.5$)$ & 78.6 \\
\hline Adjusted & $25.0(11.5 \text { to } 43.4)^{*}$ & $97.3(92.2 \text { to } 99.4)^{*}$ & 72.7 (39.0 to 93.9$)$ & 81.5 (73.8 to 87.8$)$ & 81.3 \\
\hline
\end{tabular}


after adjustment. This would suggest this simple adjustment improves the diagnostic accuracy of the Sokolow-Lyon criteria nearing that seen in normal weight subjects. In support of this, increases in Youden index (0.28 (CI 0.21 to 0.35$)$ ), positive predictive value $(0.66$ (CI 0.54 to 0.76$)$ ) and negative predictive value (0.84 (CI 0.81 to 0.86$)$ ) were seen (table 2 ).

\section{Validating the proposed adjustment}

In order to validate the proposed ECG adjustment, this was repeated in an independent cohort of 520 participants. Of these, 48 had CMR-defined LVH (9.3\%). As with the initial cohort, despite an increase in LV mass accompanying obesity $(21 \mathrm{~g}, \mathrm{p}<0.001)$, ECG voltage criteria decreased by $4 \mathrm{~mm}$ $(\mathrm{p}<0.001$, table 1$)$.

When adjusting the Sokolow-Lyon index (overweight $+4 \mathrm{~mm}$, obesity $+8 \mathrm{~mm}$ ) for the entire validation cohort, sensitivity increased significantly from $17.9(8.9-28 \cdot 7)$ to $28 \cdot 1(17 \cdot 6-$ $40 \cdot 8), \mathrm{p}<0.001$, table 2 . As with the initial cohort, large gains in sensitivity were observed in the obesity group, from 9.4 (1.1$28 \cdot 0)$ to $25 \cdot 0(11.5-43 \cdot 4)$, and also observed in the overweight group, from $8 \cdot 3(1.1-28 \cdot 0)$ to $39 \cdot 1(19.7-61 \cdot 5)$, table 2 , all $\mathrm{p}<0.001$. Again, specificity remained high even after adjustment $(>93.1 \%)$. This confirms, in a validation cohort, that this simple adjustment can substantially improve the diagnostic accuracy of the Sokolow-Lyon criteria in detecting LVH (Youden index increases from 0.12 (CI 0.02 to 0.23 ) to 0.30 (CI 0.17 to 0.42 ), ROC AUC increases from 0.60 to 0.70 ). However, due to the small sample size of non-Europeans in this study, this adjustment has not been sufficiently validated in the non-Caucasian population.

\section{DISCUSSION}

Although the Sokolow-Lyon and Cornell criteria are frequently used in clinical practice and appear widely in international guidelines, in the modern era of increasing obesity, their diagnostic accuracy is well below an acceptable level for a diagnostic screening tool. This study has shown that by incorporating BMI into the ECG algorithm by a simple adjustment, the diagnostic sensitivity can be improved without a significant decrease in specificity.

\section{The challenges of ECG screening for LVH in the modern population}

In current practice, LVH is most accurately determined by CMR, as its accuracy far exceeds that of either echocardiography or ECG. ${ }^{22}$ However, the greater availability, simplicity of operation and lower cost associated with the ECG have resulted in its continued worldwide use. However, obesity affects the surface ECG significantly, reducing voltage amplitude through a combination of leftward LV axis deviation, increased chest wall fat and increased pericardial fat. In this study, obesity was observed to reduce the sum of the R wave amplitude in V5 or 6 and the $S$ wave in $\mathrm{V} 1$ by up to $8 \mathrm{~mm}$. Indeed, we demonstrate that the sensitivity of the Sokolow-Lyon criteria is only $3.1 \%$ in obesity, with a specificity reaching $99.0 \%$. Although the specificity seems excellent, this likely reflects the fact that in obesity the degree of LVH required to generate $>35 \mathrm{~mm}$ SokolowLyon index is much greater, reducing its diagnostic power (reflected by the Youden index of 0.11). It is quite clear from this study that the Sokolow-Lyon criteria are completely inadequate to be used as a diagnostic screening test in the modern era of obesity. Although the Cornell criteria are seen here to be less vulnerable to increasing BMI, they also have poor diagnostic sensitivity $(14 \cdot 8 \%$ and $11.9 \%$, respectively).

\section{Adjusting the ECG for obesity}

It has previously been shown that the diagnostic sensitivity of the ECG can be improved by accounting for obesity. However, prior studies have either used 2D echocardiography to determine LV mass, which itself is limited in obesity ${ }^{1013}{ }^{23}$ or have used complex adjustment equations based on regression, which are unsuited to time-limited modern clinical medicine. ${ }^{24} 25$ This is the first study to use CMR to investigate the effects of both obesity and associated leftward LV axis deviation on ECG LVH criteria. We have shown that being overweight reduces the Sokolow-Lyon voltage by on average $4 \mathrm{~mm}$ and obese by $8 \mathrm{~mm}$. When using a correction factor of $+4 \mathrm{~mm}$ in overweight and $+8 \mathrm{~mm}$ in obesity, the diagnostic sensitivity of this criteria is increased (by up to $30 \%$ in overweight) and to a level that approaches that seen in normal weight. Importantly, although specificity for LVH does decrease after adjustment (by up to $5.5 \%)$, it remains excellent (92.9-97.9\%).

Given the global utilisation of this criteria as well as the worldwide increase in obesity, this finding is of significant clinical impact and should allow a substantial increase in the detection of anatomical LVH using ECG screening. As ECG-determined LVH appears in both European and US guidelines ${ }^{4}$ and is known to predict mortality, ${ }^{2} 26$ improving ECG diagnostic performance should quickly translate into significant patient benefit. However, despite these significant improvements, the sensitivity of the ECG remains poor at around $30 \%$. In the current era, this is a level that would preclude the ECG being taken up as a screening tool for LVH if presented as a novel diagnostic test.

\section{CONCLUSION}

ECG criteria for LVH severely underestimate the prevalence of anatomical LVH, especially in the setting of obesity. We propose a simple adjustment of the ECG Sokolow-Lyon criteria $(+4 \mathrm{~mm}$ in overweight, $+8 \mathrm{~mm}$ in obesity) that improves the diagnostic sensitivity for the detection of anatomical LVH without significantly decreasing the diagnostic specificity.

\section{Key messages}

What is already known on this subject?

The electrocardiogram (ECG) remains the most widely used clinical screening tool for the detection of left ventricular hypertrophy (LVH), and yet existing diagnostic criteria are increasingly insensitive due to their inaccuracy in higher body weights.

\section{What might this study add?}

Adjusting the Sokolow-Lyon index by $+4 \mathrm{~mm}$ in patients with overweight (body mass index (BMI) is $25-30 \mathrm{~kg} / \mathrm{m}^{2}$ ) and by $+8 \mathrm{~mm}$ in patients with obesity (BMI $>30 \mathrm{~kg} / \mathrm{m}^{2}$ ) improves the diagnostic accuracy for detecting LVH.

\section{How might this impact on clinical practice?}

This simple, clinically usable adjustment criterion significantly improves the power of the 12-lead ECG to detect LVH. Improving the diagnostic accuracy of the ECG should increase the number of patients identified with asymptomatic end organ damage in at-risk populations, improve risk stratification and may reduce the need for further unnecessary investigation. 
Contributors OJR, NN, SCB, SN and HW made substantial contributions to conception and design of the study. OJR, RN, VF, DH, CJH, JJR and RB acquired the MRI data. OJR, SCB, DH, NN and JJR acquired and analysed the ECG data. OJR drafted the manuscript. OJR, SM, HW and SN revised the manuscript critically for important intellectual content. All authors read and approved the final manuscript.

Funding The study was supported by grants from the British Heart Foundation (FS/08/074/26233) and Wellcome Trust (078872/Z/05/Z). SN and OJR acknowledge support from the Oxford BHF Centre of Research Excellence and the Oxford NIHR Biomedical Research Centre.

Competing interests None declared.

Ethics approval NRES Committee South Central-Oxford.

Provenance and peer review Not commissioned; externally peer reviewed.

Open Access This is an Open Access article distributed in accordance with the terms of the Creative Commons Attribution (CC BY 4.0) license, which permits others to distribute, remix, adapt and build upon this work, for commercial use, provided the original work is properly cited. See: http://creativecommons.org/ licenses/by/4.0/

\section{REFERENCES}

1 Lindholm LH, Ibsen H, Dahlöf B, et al. Cardiovascular morbidity and mortality in patients with diabetes in the Losartan Intervention For Endpoint reduction in hypertension study (LIFE): a randomised trial against atenolol. Lancet 2002;359:1004-10.

2 Kreger BE, Cupples LA, Kannel WB. The electrocardiogram in prediction of sudden-death-Framingham-study experience. Am Heart J 1987;113:377-82.

3 Taylor J. 2013 ESH/ESC guidelines for the management of arterial hypertension. Eur Heart J 2013;34:2108-9.

4 Aronow WS, Fleg JL, Pepine CJ, et al. ACCF/AHA 2011 expert consensus document on hypertension in the elderly: a report of the American College of Cardiology Foundation Task Force on Clinical Expert Consensus documents developed in collaboration with the American Academy of Neurology, American Geriatrics Society, American Society for Preventive Cardiology, American Society of Hypertension, American Society of Nephrology, Association of Black Cardiologists, and European Society of Hypertension. J Am Coll Cardiol 2011;57:2037-114.

5 Sokolow M, Lyon TP. The ventricular complex in left ventricular hypertrophy as obtained by unipolar precordial and limb leads. Am Heart J 1949;37:161-86.

6 Casale PN, Devereux RB, Kligfield P, et al. Electrocardiographic detection of left ventricular hypertrophy: development and prospective validation of improved criteria. J Am Coll Cardiol 1985;6:572-80.

7 Levy D, Labib SB, Anderson KM, et al. Determinants of sensitivity and specificity of electrocardiographic criteria for left ventricular hypertrophy. Circulation 1990;81:815-20.

8 Devereux RB, Phillips MC, Casale PN, et al. Geometric determinants of electrocardiographic left ventricular hypertrophy. Circulation 1983;67:907-11.

9 Abergel E, Tase M, Menard J, et al. Influence of obesity on the diagnostic value of electrocardiographic criteria for detecting left ventricular hypertrophy. Am J Cardiol 1996;77:739-44.
10 Okin PM, Roman MJ, Devereux RB, et al. ECG identification of left ventricular hypertrophy. Relationship of test performance to body habitus. Journal of electrocardiology 1996;29(Suppl):256-61.

11 Rosenbaum S, Crowdy JP. British Army recruits: 100 years of heights and weights. J R Army Med Corps 1992;138:81-6.

12 Rider OJ, Lewis AJ, Neubauer S. Structural and metabolic effects of obesity on the myocardium and the aorta. Obes Facts 2014;7:329-38.

13 Sugita S, Takada K, Hayano J. Influence of body composition on electrocardiographic identification of left ventricular hypertrophy in adolescents. Cardiology 1999;91:127-33.

14 Rautaharju PM, Zhou SH, Park LP. Improved ECG models for left ventricular mass adjusted for body size, with specific algorithms for normal conduction, bundle branch blocks, and old myocardial infarction. J Electrocardiol 1996;29 (Suppl):261-9.

15 Bacharova L, Chen H, Estes EH, et al. Determinants of discrepancies in detection and comparison of the prognostic significance of left ventricular hypertrophy by electrocardiogram and cardiac magnetic resonance imaging. Am J Cardiol 2015;115:515-22.

16 Rider OJ, Lewis AJ, Lewandowski AJ, et al. Obese subjects show sex-specific differences in right ventricular hypertrophy. Circ Cardiovasc Imaging 2014;8:pii: e002454.

17 Rider OJ, Nethononda R, Petersen SE, et al. Concentric left ventricular remodeling and aortic stiffness: a comparison of obesity and hypertension. Int I Cardiol 2013; 167:2989-94.

18 Banerjee R, Rial B, Holloway CJ, et al. Evidence of a direct effect of myocardial steatosis on LV hypertrophy and diastolic dysfunction in adult and adolescent obesity. JACC Cardiovasc Imaging 2015;8:1468-70.

19 Rider OJ, Lewandowski A, Nethononda R, et al. Gender-specific differences in left ventricular remodelling in obesity: insights from cardiovascular magnetic resonance imaging. Eur Heart J 2013;34:292-9.

20 Hudsmith LE, Petersen SE, Francis JM, et al. Normal human left and right ventricular and left atrial dimensions using steady state free precession magnetic resonance imaging. J Cardiovasc Magn Reson 2005;7:775-82.

21 Molloy TJ, Okin PM, Devereux RB, et al. Electrocardiographic detection of left ventricular hypertrophy by the simple QRS voltage-duration product. J Am Coll Cardiol 1992;20:1180-6.

22 Lorenz $\mathrm{CH}$, Walker ES, Morgan VL, et al. Normal human right and left ventricular mass, systolic function, and gender differences by cine magnetic resonance imaging. J Cardiovasc Magn Reson 1999;1:7-21.

23 Norman JE Jr, Levy D. Improved electrocardiographic detection of echocardiographic left ventricular hypertrophy: results of a correlated data base approach. J Am Coll Cardiol 1995;26:1022-9.

24 Norman JE Jr, Levy D. Adjustment of ECG left ventricular hypertrophy criteria for body mass index and age improves classification accuracy. The effects of hypertension and obesity. J Electrocardiol 1996;29(Suppl):241-7.

25 Okin PM, Jern S, Devereux RB, et al. Effect of obesity on electrocardiographic left ventricular hypertrophy in hypertensive patients: the losartan intervention for endpoint (LIFE) reduction in hypertension study. Hypertension 2000;35:13-18.

26 Kannel WB, Gordon T, Offutt D. Left ventricular hypertrophy by electrocardiogram. Prevalence, incidence, and mortality in the Framingham study. Ann Intern Med 1969;71:89-105. 